\title{
IDO1 Inhibitor KHK2455
}

National Cancer Institute

\section{Source}

National Cancer Institute. IDO1 Inhibitor KHK2455. NCI Thesaurus. Code C129790.

An orally available inhibitor of indoleamine 2,3-dioxygenase 1 (IDO1), with potential immunomodulating and antineoplastic activities. Upon administration, IDO1 inhibitor KHK2455 targets and binds to IDO1, an enzyme responsible for the oxidation of tryptophan into kynurenine. By inhibiting IDO1 and decreasing kynurenine in tumor cells, KHK2455 increases and restores the proliferation and activation of various immune cells, including dendritic cells (DCs), natural killer (NK) cells, and T-lymphocytes. KHK2455 also induces increased interferon (IFN) production, and causes a reduction in tumorassociated regulatory T-cells (T regs). Activation of the immune system, which is suppressed in many cancers, may inhibit the growth of IDO1-expressing tumor cells. IDO1, a cytosolic enzyme responsible for tryptophan catabolism and the conversion of tryptophan into kynurenine, is overexpressed by a variety of tumor cell types and antigen presenting cells (APCs); it plays an important role in immunosuppression. Tryptophan depletion inhibits T-lymphocyte proliferation and activation, and subsequently suppresses the immune system. 